\title{
DIFFERENT CONCEPTS OF DERIVATIVES
}

\author{
Bhuwan Prasad Ojha \\ Department of Mathematics, Apex College \\ Email Address: bhuwanp.pjha@gmail.com
}

\begin{abstract}
In this paper, different concept of derivatives with some properties has been introduced. In differential calculus, the partial derivative, directional derivative and total derivative are studied. Their generalization for Banach spaces are the Gateaux differential and Freshet derivative.
\end{abstract}

Keywords: Partial Derivative, Total Derivative, Directional Derivative, Frechet Derivative, Gateaux Derivative.

\section{Introduction}

Recall that when we discussed the interpretation of the derivative, it was the change in the function if the input were to change by one unit. G. W. Leibnitz (1646-1716), the renowned mathematician, was one of the pioneers who originated the concept of calculus. He connected the concept of derivative with the slope of the tangent to the curve at a point on it. All the problems involving rate of change can be approached using the derivative. If $y=f(x)$, then the value of $y$ is totally depends on $x$ only. If we take real world problems, there may be two or more than two independent variables affecting dependent variables. Such as quantity of production depends on a number of variables such as Labor, capital, demands of commodity, market price etc. Thus a small change in any one of the above mentioned independent variables would definitely bring some changes on dependent variable. to know this cause and effect and to find the change in dependent variable when there is a very small change in only one independent variable at a time, we use the partial derivative. Definition: Let $\mathrm{S}$ be an open set in a Euclidean space $R^{n}$, and let $\mathrm{f}: \mathrm{S} \rightarrow \mathrm{R}$ be a real valued function defined on $\mathrm{S}$. If $\mathrm{x}=\left(x_{1}, x_{2}, \ldots, x_{n}\right)$ are two points of $\mathrm{S}$ having corresponding coordinates equal except for the $k^{\text {th }}$, that is, if $x_{i \neq} c_{i}$ for $\mathrm{i} \neq \mathrm{k}$ and $x_{k \neq} c_{k}$ then we consider the limit

$$
\lim _{x_{k} \rightarrow c_{k}} \frac{f(x)-f(c)}{x_{k}-c_{k}}
$$

when the limit exists, it is called the partial derivative off with respect to $k^{\text {th }}$ coordinate and is denoted by $D_{k} f(c), \frac{\partial f}{\partial x_{k}}(\mathrm{c})$.

Partial differentiation is not really a new concept, we are merely treating $\mathrm{f}\left(x_{1}, x_{2}, \ldots, x_{n}\right)$ as a function of one variable at a time, holding the others fixed. The existence of partial derivatives with respect to each variable separately implies continuity in each variable separately, but this does not necessarily imply continuity in each variable simultaneously. We illustrate with the following example of a function of two variables:

$\mathrm{f}(\mathrm{x}, \mathrm{y})=\left\{\begin{array}{c}x+y \text { if } x=0 \text { or } y=0 \\ 1 \text { otherwise }\end{array}\right.$

The partial derivatives $D_{1} \mathrm{f}(0,0)$ and $D_{2} \mathrm{f}(0,0)$ both exists but it is not continuous at $(0,0)$. 


\section{Directional Derivative}

The partial derivative describes the rate of change of a function in the direction of each coordinate axes. There is a straight generalization, called the directional derivative, which studies the rate of change of function in an arbitrary direction. Definition: Let $\mathrm{S} \subset R^{n}$ and $\mathrm{f}: \mathrm{S} \rightarrow R^{m}$. We assume that $\mathrm{x}$ is an interior point of $\mathrm{S}$. The directional derivative of $\mathrm{f}$ at $\mathrm{x} \in \mathrm{S}$ in the direction $\mathrm{h} \in \mathrm{S}$, denoted by $f^{\prime}(\mathrm{x}$, h) and defined by the equation $f^{\prime}(x, h)=\lim _{t \rightarrow 0} \frac{f(x+t h)-f(x)}{t}$, whenever the limit on the right exist.

If $\mathrm{h}=0, f^{\prime}(\mathrm{x}, 0)$ exists and $\forall \mathrm{x} \in \mathrm{S} f^{\prime}(\mathrm{x}, 0)=0$.

If $\mathrm{h}=\left(0, \ldots, h_{k}, \ldots, 0\right)$, then $f^{\prime}(\mathrm{x}, \mathrm{h})$ is called partial derivative with respect to $\mathrm{k}$-th variable and is denoted by $D_{k} \mathrm{f}(\mathrm{x})$. If $\mathrm{f}=\left(f_{1}, f_{2}, \ldots f_{m}\right)$ then $f^{\prime}(\mathrm{x}, \mathrm{h})$ exists if and only if $f^{\prime} \mathrm{k}(\mathrm{x}, \mathrm{h})$ exists for each $\mathrm{k}=$ $1,2, \ldots, \mathrm{m}$.

In particular, when $\mathrm{h}=h_{k}, D_{k} f^{\prime}(\mathrm{x})=\left(D_{k} f_{1}^{\prime}(\mathrm{x}), D_{k} f_{2}^{\prime}(\mathrm{x})(\mathrm{x}), \ldots, D_{k} f_{m}^{\prime}(\mathrm{x})\right)$.

The directional derivative $f^{\prime}(\mathrm{x}, \mathrm{h})$, if exists in every direction, is homogeneous in h, i.e. $\forall \mathrm{x} \in \mathrm{X} \forall \mathrm{a}$ $\in \mathrm{R}, \operatorname{Df}(\mathrm{x}, \mathrm{ah})=\mathrm{aDf}(\mathrm{x}, \mathrm{h})$, however it is not additive and linear.

Thus, one of the important property of a derivative, linearity, is absent in the directional derivative. Besides that the existence of all directional derivatives at a point fails to imply continuity at that point. For example let $\mathrm{f} f(x, y)=\left\{\begin{array}{cc}\frac{x y^{2}}{x^{2}+y^{4}}, & \text { if } x \neq 0 \\ 0, & x=0\end{array} f^{\prime}(0, \mathrm{~h})\right.$ exists for all $\mathrm{h}$, but $\mathrm{f}$ is not continuous at $(0,0)$.

\section{Total derivative}

The existence of all directional derivatives at a point fails to imply continuity at that point. For this reason, directional derivatives, like partial derivatives, are a somewhat unsatisfactory extension of the one dimensional concept of derivative. We turn now to more suitable generalization, called Total derivative, which implies continuity also.

Definition: The function $\mathrm{f}: \mathrm{S} \subset R^{n} \rightarrow R^{m}$ is said to be differential at $\mathrm{c}$ if there exists a linear function $T_{c}: R^{n} \rightarrow R^{m}$ such that $\mathrm{f}(\mathrm{c}+\mathrm{v})=\mathrm{f}(\mathrm{c})+T_{c}(\mathrm{v})+\|\mathrm{v}\| E_{c}(\mathrm{v})$, where $E_{c}(\mathrm{v}) \rightarrow 0$ as $\mathrm{v} \rightarrow 0$. The linear function $T_{c}$ is called the total derivative of $\mathrm{f}$ at $\mathrm{c}$.

Theorem 3.1: Assume that $\mathrm{f}$ is differential at $\mathrm{c}$ with total derivative $T_{c}$. Then the directional derivative $f^{\prime}(\mathrm{c}, \mathrm{u})$ exists for every $\mathrm{u}$ in $f^{\prime}$ and we have $T_{c}(\mathrm{u})=f^{\prime}(\mathrm{c}, \mathrm{u})$.

\section{Gateaux Derivative}

Definition: Let $f$ be a mapping on an open subset $U$ of a normed space $\mathrm{X}$ into normed space $\mathrm{Y}$. We say that $f$ is Gateaux differentiable at $x \in U$ if

1. The directional at $\mathrm{x}$ exists in all directions, i. e., $\forall \mathrm{h} \in \mathrm{X} \backslash\{0\} \mathrm{Df}(\mathrm{x}, \mathrm{h})=\lim _{t \rightarrow 0} \frac{f(x+t h)}{t}$ exists.

2. $\quad \operatorname{Df}(\mathrm{x}, \mathrm{h})$ is linear in $\mathrm{h}$.

The operator $\operatorname{Df}(\mathrm{x}, \mathrm{h}) \in \mathrm{Y}$ is called the Gateaux differential of $\mathrm{f}$ at $\mathrm{x}$ in the direction $\mathrm{h}$. One of the epochal properties of Gateaux derivative is that, there is not a single Gateaux differential at each point. Rather, at each point $\mathrm{x}$ there is a Gateaux differential for each direction $\mathrm{h}$. For example, in one dimension, there are two Gateaux differentials for every $\mathrm{x}$; one directed "forward", the other backward. In two of more dimensions, there are infinitely many Gateaux differentials at each point. The Gateaux differential is a one- dimensional calculation along a specified direction $\mathrm{h}$ so that ordinary one dimensional calculation can be used to compute it. Thus it is easy to compute a Gateaux differential even when the space is an infinite dimensional. 
Definition: Let $(\mathrm{X},\|\|$.$) be a normed space. We say that the norm function \mathrm{f}=\|$.$\| is Gateaux$ differentiable for all $\mathrm{x} \neq 0$ if $\forall \mathrm{h} \in \mathrm{X} \backslash\{0\} \operatorname{Df}(\mathrm{x}, \mathrm{h})=\lim _{t \rightarrow 0} \frac{\|x+t h\|-\|x\|}{t}(\mathrm{t} \in \mathrm{R})$ exists. It is notice that norm is not differential at origin, because

$\operatorname{Df}(0, \mathrm{~h})=\lim _{t \rightarrow 0} \frac{\|0+t h\||-||0| \mid}{t}=\lim _{t \rightarrow 0} \frac{|t|}{t}\|\mathrm{~h}\|$

Clearly this does not exists.

The norm may or may not be differentiable at a point other than the origin. For example, the norm $\|$. $\|_{p}$ defined on $R^{n}$ is differentiable at all points other than the origin if $\mathrm{p} \in(1, \infty)$; however, this is not the case if $\mathrm{p}=1$ or $\mathrm{p}=\infty$.

Theorem 4.1: Let $(X,\|\|$,$) be a real normed space. If f=\|$.$\| is differential at x \neq 0$. Then $\operatorname{Df}(x,$.$) is a$ real linear functional on $\mathrm{X}$ with $\|\mathrm{Df}(\mathrm{x},)\|=$.1 .

Theorem 4.2: The Gateaux derivative of a mapping $\mathrm{f}$ is unique provided it exists. Theorem 4.3: A sub linearfunctional $\mathrm{p}$ on a real vector space $\mathrm{X}$ is linear if and only if

$\mathrm{p}(-\mathrm{x})=-\mathrm{p}(\mathrm{x})$ for all $\mathrm{x} \in \mathrm{X}$.

Theorem 4.4: For fixed $\mathrm{x} \in \mathrm{X}$, the mapping $\mathrm{Df}(\mathrm{x},$.$) is a real linear functional on \mathrm{X}$.

\section{Frechet Derivatives}

The concepts of the Gateaux differential did not require any notion of convergence on the domain space, and this can led to some "unusual" properties. For example $\mathrm{f}: R^{2} \rightarrow \mathrm{R}$ defined by $\mathrm{f}(x, y)=$ $\left\{\begin{array}{cl}\frac{x^{4} y}{x^{6}+y^{3}}, & \text { if }(x, y) \neq 0 \\ 0, & \text { if } \quad(x, y)=0\end{array}\right.$,

has gateaux differential at origin but fails to be continuous there. In order to make sure that differentiable functions are continuous we now introduce a stronger concept of derivative. In One dimensional case, a function $\mathrm{f}$ with a derivative at $\mathrm{x}$ can be approximated by a linear polynomial. In fact if $f^{\prime}(\mathrm{x})$ exists, let $E_{x}(\mathrm{~h})$ denote the difference

$E_{x}(\mathrm{~h})=\frac{\mathrm{f}(\mathrm{x}+\mathrm{h})-\mathrm{f}(\mathrm{x})}{h}-f^{\prime}(\mathrm{x})$ if $\mathrm{h} \neq 0$ and let $E_{x}(\mathrm{~h})=0$.

Then we have $f(x+h)=f(x)+f^{\prime}(x) h+h E_{x}(h)$. This is called the first order Taylor formula for approximating $\mathrm{f}(\mathrm{x}+\mathrm{h})-\mathrm{f}(\mathrm{x})$ by a linear function $f^{\prime}(\mathrm{x}) \mathrm{h}$. The error committed is $\mathrm{h} E_{x}(\mathrm{~h})$. We see that $E_{x}(\mathrm{~h}) \rightarrow 0$ as $\mathrm{h} \rightarrow 0$. The error $E_{x}(\mathrm{~h})$ is said to be of smaller order than $\mathrm{h}$ as $\mathrm{h} \rightarrow 0$. Thus, $\mathrm{h} E_{x}(\mathrm{~h})=\mathrm{o}$ (h).

Definition: Let $\mathrm{f}$ be a function on an open subset $\mathrm{U}$ of a normed space $\mathrm{X}$ into the normed space $\mathrm{Y}$. We say $\mathrm{f}$ is Frechet differentiable at $\mathrm{x} \in \mathrm{U}$ if there is a bounded linear operator $T_{x}: \mathrm{X} \rightarrow \mathrm{Y}$ such that $\mathrm{f}(\mathrm{x}+$ $\mathrm{y})=\mathrm{f}(\mathrm{x})+T_{x} \mathrm{y}+\|\mathrm{y}\| E_{x}(\mathrm{y})$, where $E_{x}(\mathrm{y}) \rightarrow 0$ as $\mathrm{y} \rightarrow 0$.

In this case, $T_{x}(\mathrm{y}) \in \mathrm{Y}$ is called the Frechet differential of $\mathrm{f}$ at $\mathrm{x}$. It is denoted by $f^{\prime}(\mathrm{y})=T_{x} \mathrm{y}$. The operator $T_{x}=f^{\prime}(\mathrm{x})$ is called Frechet derivative of $\mathrm{f}$ at $\mathrm{x}$.

Theorem 5.1: If $\mathrm{f}$ is a bounded in a neighborhood of $\mathrm{x}$ and if a linear map $T_{x}$ has the property

$\forall \mathrm{y} \in \mathrm{X} \quad \lim _{y \rightarrow 0} \frac{f(x+y)-f(x)-T_{x}(y)}{\|y\|}=0$. Then $T_{x}$ is a bounded linear map; in other words, $T_{x}$ is the Frechet derivative of $\mathrm{f}$ at $\mathrm{x}$. 
Theorem 5.2: Let $\mathrm{X}, \mathrm{Y}$ be normed spaces. If $\mathrm{f}: \mathrm{X} \rightarrow \mathrm{Y}$ is differentiable at $x_{0} \in \mathrm{X}$, then

1. The derivative $f^{\prime}\left(x_{0}\right)$ of $f$ at $x_{0}$ is unique.

2. $\mathrm{f}$ is continuous at $x_{0}$.

Theorem 5.3: If an operator has the Frechet derivative at a point, then it has the Gateaux derivative at that point and both derivatives are equal.

It is noted that a function which is Frechet differentiable at a point is continuous there but this is not the case for Gateaux differentiable functions even in finite dimensions. The definition of differentiability depends not only the norms but on the topologies of $\mathrm{X}$ and $\mathrm{Y}$ only. That is, if for example $\|.\|_{1}$ and $\|.\|_{2}$ are two equivalent norms on $\mathrm{X}$ then $\mathrm{f}$ is differentiable at $\mathrm{x}$ in $\|.\|_{1}$ if and only if $\mathrm{f}$ is in $\|.\|_{2}$ and the differential is the same.

Theorem 5.4: Let $(X,\|\|$.$) be a real normed space. If \mathrm{f}=\|$.$\| is differentiable at \mathrm{x} \neq 0$, then $\operatorname{Df}(\mathrm{x},$.$) is a$ real linear functional on $\mathrm{X}$ with $\|\mathrm{D} f(\mathrm{x},)\|=$.1 and moreover, the norm is differentiable at $\lambda \mathrm{x}$ for $\lambda>0$ and $\|\lambda \times\|^{\prime}=\|\mathrm{x}\|^{\prime}$.

Theorem 5.5: Let $\mathrm{f}$ be convex function defined on an open convex subset $\mathrm{U}$ of a Banach space $\mathrm{X}$ that is continuous at $\mathrm{x} \in \mathrm{U}$. Then $\mathrm{f}$ is Frechet differentiable at $\mathrm{x}$ if and only if

$\lim _{t \rightarrow 0} \frac{f(x+t h)+f(x-t h)-2 f(x)}{t}=0$ uniformly for $\mathrm{h} \in S_{x}$.

Theorem 5.6: (Smulian Lemma) Let(X, \|.||) be a Banach space and let $\mathrm{x} \in S_{x}$. Then the following statements are equivalent.

1. $\|$.$\| is Frechet differentiable at \mathrm{x}$.

2. For all sequences $\left\{f_{n}\right\},\left\{g_{n}\right\} \subset \mathrm{S}\left(X^{*}\right) \lim _{n \rightarrow \infty} f_{n(x)}=\lim _{n \rightarrow \infty} g_{n(x)}=1 \Rightarrow \lim _{n \rightarrow \infty}|| f_{n-} g_{n} \|=0$.

3. $\forall\left\{f_{n}\right\} \subset \mathrm{S}\left(X^{*}\right) \lim _{n \rightarrow \infty} f_{n(x)}=1 \Rightarrow\left\{f_{n}\right\}$ convergent in $\mathrm{S}\left(X^{*}\right)$.

\section{References}

1. Apostol, T.M., "Mathematical Analysis", 2nd ed. California Institute of Technology, 346350 .

2. Behmard, D. and Nayeri, E., "Introduction of Freshet Derivative and Gateaux Derivative."

3. Chen, W. and Ditzian, Z., "Mixes and Directional Derivatives", Proceeding of AMS, vol108, (177-185), 1990.

4. Rode. G. and Simons, S., "Directional Derivative and Variational Inequalities", Journal of Mathematical Analysis and Applications, vol. 95, (284-296), 1983.

5. Long, K., “Gateaux Differentials and Frechet Derivativs”, AMS, (12-26), 2009.

6. Spova, M., “Conditions for Differentiability in Linear Topological Spaces”, Czechoslovk, Math. J.16, (339-362), 1966.

7. Gonzalez-Gaxiola, O., "A note on the Derivative of Frechet and gateaux", Mathematical Sciences, vol.3, (941-947), 2009. 